TRANSACTIONS OF THE

AMERICAN MATHEMATICAL SOCIETY

Volume 361, Number 9, September 2009, Pages 4901-4925

S 0002-9947(09)04810-7

Article electronically published on April 13, 2009

\title{
DISTRIBUTIONAL CHAOS REVISITED
}

\author{
PIOTR OPROCHA
}

Dedicated to Professor Jaroslav Smital on the occasion of his 65th birthday.

\begin{abstract}
In their famous paper, Schweizer and Smítal introduced the definition of a distributionally chaotic pair and proved that the existence of such a pair implies positive topological entropy for continuous mappings of a compact interval. Further, their approach was extended to the general compact metric space case.

In this article we provide an example which shows that the definition of distributional chaos (and as a result Li-Yorke chaos) may be fulfilled by a dynamical system with (intuitively) regular dynamics embedded in $\mathbb{R}^{3}$. Next, we state strengthened versions of distributional chaos which, as we show, are present in systems commonly considered to have complex dynamics.

We also prove that any interval map with positive topological entropy contains two invariant subsets $X, Y \subset I$ such that $\left.f\right|_{X}$ has positive topological entropy and $\left.f\right|_{Y}$ displays distributional chaos of type 1, but not conversely.
\end{abstract}

\section{INTRODUCTION}

Since its origins in the fundamental paper by $\mathrm{Li}$ and Yorke 34 the existence of a pair of points which are proximal but not asymptotic (a so-called Li-Yorke pair) has attracted many researchers. It was a long standing problem, what are the relations between positive topological entropy and the existence of Li-Yorke pairs. A theorem of Misiurewicz 39] which characterizes positive topological entropy of interval maps in terms of topological horseshoes provides a tool for finding the answer. This result gave a tool to prove that positive topological entropy implies the existence of a Cantor set which is a scrambled set at the same time 30] (it is remarkable that this statement holds in general compact metric spaces [10]). It was much more demanding to show that there exists an interval map with zero topological entropy and an uncountable scrambled set; however, both results were obtained at almost the same time [56] (later, strict conditions when such constructions are possible were given [22]).

Various extensions of the definition by Li and Yorke were developed, e.g. dense chaos [48, 60, generic chaos [59] or extreme chaos [14] (see survey paper [11] for a discussion). Results cited above extend the definition of Li and Yorke by some additional assumptions which specify (in the topological sense) how the scrambled

Received by the editors October 17, 2007.

2000 Mathematics Subject Classification. Primary 37B99; Secondary 37D45, 37B10, 37B20.

Key words and phrases. Distributional chaos, chaotic pair, Li-Yorke chaos.

(C)2009 American Mathematical Society

Reverts to public domain 28 years from publication 
set is placed in the space. Another approach is to strengthen the definition of a Li-Yorke pair by same statistical assumptions.

In the paper [52, B. Schweizer and J. Smítal introduced a notion of distributional chaos (it was called strong chaos there). Their fundamental observation was that in the case of a map $f$ from a compact interval into itself the existence of at least one distributionally chaotic pair (in a weak sense, called $D C 3$ ) implies positive topological entropy for $f$. These results, combined with the characterization provided by $\mathrm{S}$. Li in 33 show that the existence of distributionally chaotic pairs forces a very strong chaotic behavior in the case of one-dimensional dynamics. Strictly speaking, distributional chaos, positive topological entropy, $\omega$-chaos and the existence of an infinite invariant subset on which $f$ exhibits chaos in the sense of Devaney are all equivalent properties in the case of interval maps (i.e. each of these kinds of chaos implies each other). We refer to [51] as a very nice survey on distributional chaos (and its measurement) in the case of a compact interval.

The equivalence of different kinds of chaos is no longer valid when a general compact metric space is considered (see [57, 6] or [44] for a discussion). It is also known that in the case of interval maps, the existence of a chaotic pair (in the $D C 3$ sense) implies that there exists an uncountable set such that each pair of its distinct points is distributionally chaotic (in the sense of $D C 1$ ). Such a set, if it exists, is usually called distributionally scrambled.

Recently, it was observed that it is possible to state three nonequivalent definitions of a distributionally chaotic pair and, as a result, to define three nonequivalent versions of distributional chaos for maps on compact metric spaces: $D C 1, D C 2$ and $D C 3$ (see [8] for definitions). The strongest among these definitions is $D C 1$ with an uncountable distributionally scrambled set. In this paper we focus our attention to distributional chaos of type 1 , so please keep in mind that distributional chaos always means distributional chaos of type 1 if not differently stated.

The idea behind a distributional pair (of type 1) is the following: when we look at trajectories of given points from one time perspective, then the frequency of iterations during which points are close to each other tends to 1 , but when the time perspective is changed it seems that their iterations are separated from one another almost all the time. Then there is some kind of statistical unpredictability in their motion (and obviously a distributionally chaotic pair generalizes the Li and Yorke approach). This is why we are likely to conclude chaotic behavior if we are able to find an uncountable distributionally scrambled set. In section 3 we prove that this is somehow misleading: distributional chaos with its standard definition may be present in a dynamical system with a "regular" motion. When in dimension one, we do not have to care too much about the definition of distributional chaos, because the existence of at least one chaotic pair (in the weakest sense DC3) implies positive topological entropy [52] or even much more complex dynamics [7]. An example from Section 3 highlights the fact that, in general metric spaces, we have to control not only the frequency but also the amplitude of changes in the motion of pairs.

The main idea of this paper is to modify the definition of distributionally scrambled set in a way that it becomes significant from the topological point of view, that is, to avoid its concentration on small sets (in the topological sense). Our exposition cannot be complete. For example, we will not consider a measure-theoretic approach. Let us only mention some recent advances in that field. One of the first attempts to construct chaotic sets (in the sense of the Li and Yorke definition) 
which are significant from the measure-theoretic point of view were taken in [54, where the scrambled set $S \subset I$ with full outer Lebesgue measure was constructed. Further, functions with scrambled sets of positive Lebesgue measure were obtained in [31] and [55, and finally functions Li-Yorke chaotic almost everywhere were presented in [14] and [0]. Recently, it was proved in [5] that the same is possible for distributional chaos (even for uniform distributional chaos; the definition is slightly different there, however, and it follows from the proof that the main results remain true with the stronger definition of uniform distributional chaos presented in our paper). A combination of distributional chaos with the measurement of a distributionally scrambled set may be another (parallel) approach to strengthen this definition, because the scrambled set obtained in Section 3 has zero Lebesgue measure. Another reasonable approach is to look at the Hausdorff dimension. Obviously, the distributionally scrambled set from [5] has full Hausdorff dimension, which cannot be said about the distributionally scrambled set constructed in Section 3 (however it is a perfect set which means that Cantor-like constructions are not enough in general).

The paper is organized as follows. Section 3 contains an example which motivates proposed extensions of the definition of distributional chaos. In Section 4 we make such extensions, in a way which, in our opinion, avoids defining as chaotic systems which are not. The next section highlights relations between distributional chaos and proximality. Section 6 contains a construction of an uncountable family consisting of minimal subsystems of the full shift which exhibit uniform distributional chaos of type 1 (or the even stronger property introduced in Definition 10 under the name transitive distributional chaos). In Section 7 we discuss a modification of Rafał Pikuła's example which will allow us to approximate the topological entropy of interval maps via subsystems without DC1 pairs. Section 8 combines results from the previous two sections. We show there that any interval map $f$ with positive topological entropy contains two disjoint subsets $X, Y \subset I$ such that $h_{\text {top }}\left(\left.f\right|_{X}\right)=(1-\varepsilon) h_{\text {top }}(f), h_{\text {top }}\left(\left.f\right|_{Y}\right)=0$, there is no DC1 pair for $f$ in $X$ and $Y$ contains an uncountable distributionally scrambled set of type 1 for $\left.f\right|_{Y}$ (see Theorem (35). In the last section we state a few open problems for further research.

\section{BASIC DEFINITIONS}

Let $(X, d)$ be a compact metric space and let $f: X \rightarrow X$ be continuous. By a dynamical system we mean a pair $(X, f)$. A point $y \in X$ is said to be an $\omega$-limit point of a point $x$ if it is an accumulation point of the sequence $x, f(x), f^{2}(x), \ldots$. The set of all $\omega$-limit points of $x$ is called the $\omega$-limit set of $x$ and denoted $\omega(x, f)$. A point $x$ is said to be periodic if $f^{n}(x)=x$ for some $n \geq 1$ and is said to be recurrent if $x \in \omega(x, f)$. A subset $M$ of $X$ is minimal if it is closed, nonempty, invariant (i.e. $f(M) \subset M)$ and contains no proper subset with these three properties. It is well known that if a nonempty closed set $M \subset X$ is minimal, then the orbit of every point of $M$ is dense in $M$ (the converse implication holds if there are no isolated points in $X$ ). We recall that a point $x$ is called minimal or almost periodic if it belongs to a minimal set. We will denote by $\operatorname{Per}(f), \mathrm{A}(f)$ and $\operatorname{Rec}(f)$ the sets of periodic, almost periodic and recurrent points respectively. In the case that all of $X$ is minimal we say that $f$ is a minimal system.

By $\mathrm{Orb}^{+}(x)$ we denote the set $\mathrm{Orb}^{+}(x)=\left\{x, f(x), f^{2}(x), \ldots\right\}$ and call it the positive orbit of point $x$. 
We call a point $x$ nonwandering if for any neighborhood $U$ of $x$ there exists $n>0$ such that $f^{n}(U) \cap U \neq \emptyset$. The set of all nonwandering points is said to be the nonwandering set and is denoted by $\Omega(f)$.

We recall that $f$ is (topologically) transitive if for any two nonempty open sets $U, V \subset X$ there exists $n>0$ such that $f^{n}(U) \cap V \neq \emptyset$ and is (topologically) weakly mixing if for any three nonempty open sets $U, V, W \subset X$ there exists $n>0$ such that $f^{n}(W) \cap V \neq \emptyset$ and $f^{n}(W) \cap U \neq \emptyset$ (see [9] for a list of equivalent conditions).

The topological entropy of a dynamical system $(X, f)$ acting on a compact metric space will be denoted $h_{t o p}(f)$. In the case of a compact metric space there are several equivalent definitions of topological entropy. We do not recall these definitions as they are well known; see, for instance, books [19, 37.

Let $X$ and $Y$ be compact metric spaces and let $f: X \rightarrow X$ and $g: Y \rightarrow Y$ be continuous maps. If there is a continuous onto map $\phi: X \rightarrow Y$ with $\phi \circ f=g \circ \phi$, we will say that $f$ and $g$ are semiconjugate (by $\phi$ ). The map $\phi$ is called a semiconjugacy (between $f$ and $g$ ) or a factor map, the map $g$ is called a factor of $f$ and the map $f$ is called an extension of $g$.

2.1. Li-Yorke chaos. For a dynamical system $(X, f)$ acting on a compact metric space $(X, d)$ and for any $\varepsilon>0$ we define the following sets:

$$
\begin{aligned}
C_{1}(f) & =\left\{(x, y) \in X^{2}: \liminf _{n \rightarrow+\infty} d\left(f^{n}(x), f^{n}(y)\right)=0\right\}, \\
C_{2}(f) & =\left\{(x, y) \in X^{2}: \limsup _{n \rightarrow+\infty} d\left(f^{n}(x), f^{n}(y)\right)>0\right\}, \\
C_{2}(f, \varepsilon) & =\left\{(x, y) \in X^{2}: \limsup _{n \rightarrow+\infty} d\left(f^{n}(x), f^{n}(y)\right)>\varepsilon\right\}, \\
C(f) & =C_{1}(f) \cap C_{2}(f), \\
C(f, \varepsilon) & =C_{1}(f) \cap C_{2}(f, \varepsilon) .
\end{aligned}
$$

A function $f$ is called chaotic in the sense of Li and Yorke (see 34 and 32 for a discussion) if there is an uncountable set $S$ (a so-called scrambled set) such that $C(f) \supset S \times S \backslash \Delta_{X}$ where $\Delta_{X}=\{(x, x): x \in X\}$. Motivated by this definition, L. Snoha proposed to call a map $f$ densely $\varepsilon$-chaotic if the set $C(f, \varepsilon)$ is dense in $X^{2}$ (originally the definition was stated for $I^{2}$ ). We remark that $f$ exhibits dense $\varepsilon$-chaos iff there is a dense scrambled set $S$ such that $S \times S \subset C(f, \varepsilon) \cup \Delta_{X}$ (see [50, p. 1693] for more detailed comments).

Let us recall (see [20]) that a map $f$ has sensitive dependence on the initial conditions if there exists $r>0$ such that for every $x \in X$ and every $\varepsilon>0$, we can find a point $y \in X$ such that $d(x, y)<\varepsilon$ and $d\left(f^{m}(x), f^{m}(y)\right)>r$ for some integer $m>0$. Obviously, any map with dense $\varepsilon$-chaos has sensitive dependence on the initial conditions (see [1, Thm. 3.4]).

2.2. Distributional chaos. We define a function $\xi: X \times X \times \mathbb{R} \times \mathbb{N} \longrightarrow \mathbb{N}$ by:

$$
\xi(x, y, t, n)=\#\left\{i: d\left(f^{i}(x), f^{i}(y)\right)<t, 0 \leq i<n\right\},
$$

where $\# A$ denotes the cardinality of the set $A$. By means of $\xi$ we define the following two functions:

$$
F_{x y}(t)=\liminf _{n \rightarrow \infty} \frac{1}{n} \xi(x, y, t, n), \quad F_{x y}^{*}(t)=\limsup _{n \rightarrow \infty} \frac{1}{n} \xi(x, y, t, n) .
$$


Both functions $F_{x y}$ and $F_{x y}^{*}$ are nondecreasing, $F_{x y}(t)=F_{x y}^{*}(t)=0$ for $t<0$ and $F_{x y}(t)=F_{x y}^{*}(t)=1$ for $t>\operatorname{diam} X$. Functions $F_{x y}$ and $F_{x y}^{*}$ are called lower and upper distribution functions respectively.

In this paper we will usually refer to the strongest definition of distributional chaos (so-called $D C 1$ with an uncountable distributionally scrambled set [8]); however, we recall all three possibilities known from the literature.

Definition 1. A pair of points $x, y \in X$ is called distributionally chaotic of type 1 (DC1 for short) if

(1) $F_{x y}(s)=0$ for some $s>0$,

(2) $F_{x y}^{*}(t)=1$ for all $t>0$.

If condition (11) is replaced by $F_{x y} \prec F_{x y}^{*}\left(f \prec g\right.$ means that $\int_{-\infty}^{\infty} g(t)-f(t) d t>$ 0 ), then we obtain the definition of $D C 2$ and if additionally condition (2) is omitted, then we obtain a weaker version denoted $D C 3$. Note that in the case of $D C 2$ the condition $F_{x y} \prec F_{x y}^{*}$ is equivalent to the fact that $F_{x y}(s)<1$ for some $s>0$.

A set containing at least two points is called a distributionally scrambled set of type $k$ for $f$ if any pair of its distinct points is distributionally chaotic of type $k$, where $k \in\{1,2,3\}$.

Definition 2. Let $f$ be a continuous self-map of a compact metric space. We say that $f$ is distributionally chaotic of type $k \in\{1,2,3\}$ if there exists an uncountable distributionally scrambled set of type $k$ for $f$.

Sometimes, the definition of distributional chaos is strengthened in the following way (see [5): distributional chaos of type 2 is uniform if there exists a distribution function $F$ such that $F_{x y} \leq F \leq F_{x y}^{*}$ for all distinct $x, y \in D$, where $D$ is a distributionally scrambled set of type 2 . We state a similar definition in the case of distributional chaos of type 1 . It is an analogue of $\delta$-scrambled sets defined in the case of Li-Yorke chaos.

Definition 3. Let $f$ be distributionally chaotic of type 1 and let $D$ be a distributionally scrambled set for $f$. If there exists $\varepsilon>0$ such that $F_{x y}(\varepsilon)=0$ for any distinct points $x, y \in D$, then the distributional chaos is said to be uniform.

Remark 4. The definition of distributional chaos was introduced in [52] for the first time (under the name strong chaos). This definition was presented for interval maps as a condition equivalent with positive topological entropy.

2.3. Symbolic spaces. Let $\mathcal{A}$ be any finite set (alphabet) and let $\mathcal{A}^{*}$ denote the set of all finite words over $\mathcal{A}$. The set $\mathcal{A}^{*}$ with the concatenation of words forms a free monoid with the minimal set of generators $\mathcal{A}$. The unit element of concatenation, denoted by $\lambda$, is called the empty word. For any word $w \in \mathcal{A}^{*}$ we denote by $|w|$ the length of $w$, that is, the number of letters which form this word. By $|w|_{a}$ is denoted the number of occurrences of the letter $a$ in $w$. Two-letter alphabets will be denoted $\Sigma=\{0,1\}$. Let $w=w_{1} \ldots w_{n} \in \Sigma^{*}$. By $\bar{w}$ we mean the negation of $w$, that is, $\bar{w}=\overline{w_{1}} \overline{w_{2}} \ldots \overline{w_{n}}$. For example, if $w=01001$, then $\bar{w}=10110$.

An infinite word is a mapping $w: \mathbb{N} \longrightarrow \mathcal{A}$; hence it is an infinite sequence $w_{1}, w_{2}, \ldots$ where $w_{i} \in \mathcal{A}$ for any $i \in \mathbb{N}$. The set of all infinite words over the alphabet $\mathcal{A}$ is denoted by $\mathcal{A}^{\omega}$. If $x \in \mathcal{A}^{\omega}$ and $i \leq j$ are integers, then we denote $x_{[i, j]}=x_{i} x_{i+1} \ldots x_{j}$ and $x_{[i, j)}=x_{[i, j-1]}$. To be able to consider continuous maps we have to introduce a topology. The set $\mathcal{A}$ is given the discrete topology and $\mathcal{A}^{\omega}$ 
is endowed with the product topology. This topology is metrizable and may be equivalently defined by the following metric. For any $x, y \in \mathcal{A}^{\omega}$ put

$$
d(x, y)=\left\{\begin{array}{l}
2^{-(k+1)} \text { if } x \neq y \\
0 \text { otherwise }
\end{array}\right.
$$

where $k$ is the length of the maximal common prefix of $x$ and $y$. Now define a shift operation $\sigma_{\mathcal{A}}: \mathcal{A}^{\omega} \rightarrow \mathcal{A}^{\omega}$ by

$$
\left(\sigma_{\mathcal{A}}(x)\right)_{i}=x_{i+1} .
$$

The pair $\left(\mathcal{A}^{\omega}, \sigma_{\mathcal{A}}\right)$ is said to be the full shift over $\mathcal{A}$. We will usually simply write $\sigma$ instead of $\sigma_{\mathcal{A}}$.

If we consider the space ${ }^{\omega} \mathcal{A}^{\omega}$ of bi-infinite words (i.e. sequences $\mathbb{Z} \rightarrow \mathcal{A}$ ), then we may build an analogous theory of shift spaces. The main difference is that $\sigma:{ }^{\omega} \mathcal{A}^{\omega} \rightarrow{ }^{\omega} \mathcal{A}^{\omega}$ is invertible and $d(x, y)=2^{-(k+1)}$, where $k \in \mathbb{N}$ is the maximal integer such that $x_{[-k, k]}=y_{[-k, k]}($ when $x \neq y)$. We will think of elements $x \in{ }^{\omega} \mathcal{A}^{\omega}$ as being bilateral sequences $x=\ldots x_{-2} x_{-1} \dot{x}_{0} x_{1} x_{2} \ldots$, with the dot marking the "central position" of the sequence $x$.

2.4. Recurrence index. Let $X$ be a closed $\sigma$-invariant subset of $\mathcal{A}^{\omega}$. We define the set $B_{n}(X)$ of $n$ admissible words for $X$ by:

$$
B_{n}(X)=\left\{x_{[i, i+n)} \in \mathcal{A}^{*}: x \in X, i \in \mathbb{N}\right\} .
$$

Let $w \in \mathcal{A}^{*}$. The set of all subwords of $w$ with the length $n$ ( $n$-subwords) is denoted by

$$
S_{n}(w)=\left\{u \in B_{n}\left(\mathcal{A}^{\omega}\right): \exists v_{1}, v_{2} \in \mathcal{A}^{*}, w=v_{1} u v_{2}\right\} .
$$

We may extend canonically the definition of $S_{n}$ to the case $x \in \mathcal{A}^{\omega}$; i.e., given $x \in \mathcal{A}^{\omega}$ we define:

$$
S_{n}(x)=\left\{u \in B_{n}\left(\mathcal{A}^{\omega}\right): \exists i, u=x_{[i, i+n)}\right\} .
$$

The least integer $m$ (if it exists) such that for any $w \in B_{m}(X)$ it follows that $S_{n}(w)=S_{n}(x)$ is called the $n$-th recurrency index of $x$ in $X$ and is denoted $R(n, x, X)$. If such $m$ do not exist, we put $R(n, x, X)=+\infty$. In the case that $X=\operatorname{cl}\left(\mathrm{Orb}^{+}(x)\right)$ we will simply write $R(n, x)$ instead of $R\left(n, x, \mathrm{cl}\left(\mathrm{Orb}^{+}(x)\right)\right)$, where $\operatorname{cl}(A)$ denotes the closure of the set $A$. In other words, $R(n, x)=m$ if each $m$-subword of $x$ contains every $n$-subword of $x$. Let us recall some facts on recurrence indices.

Theorem 5 ([41, Thm. 7.2]). Let $x \in \mathcal{A}^{\omega}$. The following conditions are equivalent:

(1) $x$ is a minimal point,

(2) $R(n, x)<+\infty$ for all positive integers $n$.

Theorem 6 (41, Thm. 7.1]). Let $M \subset \mathcal{A}^{\omega}$ be a minimal set. In this case $R(n, x)=$ $R(n, y)$ for any $x, y \in M$ and $n \in \mathbb{N}$.

Theorem 7 (41, Thm. 9.3]). Let $x \in \mathcal{A}^{\omega}$ and let $w \in B_{s}\left(\operatorname{cl}\left(\operatorname{Orb}^{+}(x)\right)\right)$ for some $s>0$. Suppose that there exists $r>1$ such that $w^{r} \in B_{r s}\left(\operatorname{cl}\left(\mathrm{Orb}^{+}(x)\right)\right)$ and $\sigma^{s}(x) \neq x$. In this case $R(s+1, x)>s r$. 


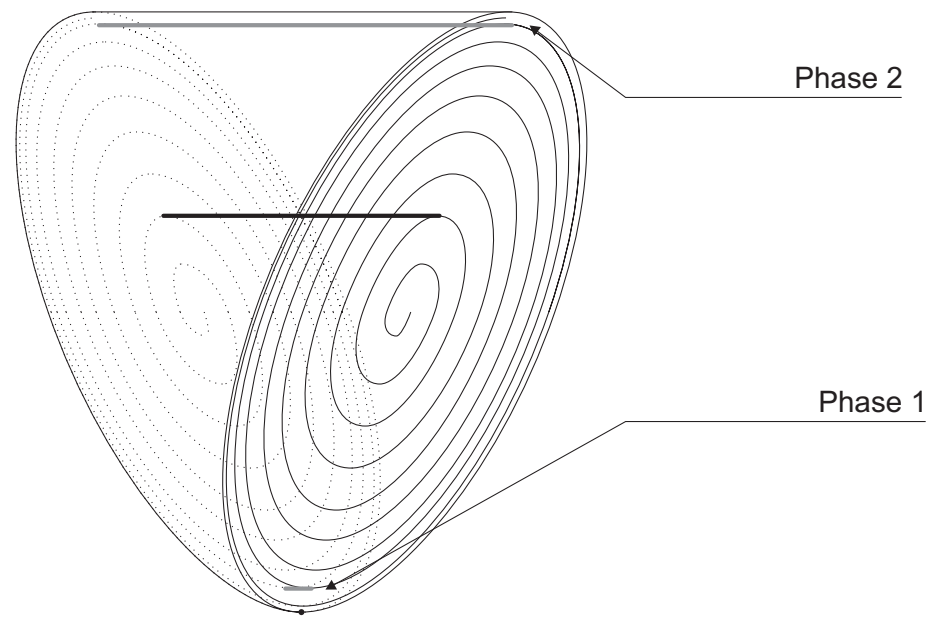

Figure 1. Visualization of the space $X$ from Example 8

\section{Fundamental observation}

In this section we will construct a dynamical system which is distributionally chaotic of type 1, but the trajectories of points are "regular" from the point of view of the qualitative theory of dynamical systems. The constructed system may be naturally considered as discretization of a continuous flow (see Fig. 1); the positive limit set of each point is either a fixed point or a circle (consisting of fixed points) contained in the boundary of the phase space. Namely, this system is an extension of a flow on the unit disc with repelling fixed point in the center and attracting circle in the boundary. The qualitative behavior of the system may be well understood from its phase portrait. There is no doubt about its asymptotic properties, periodic points, etc. There is no "mixing" of trajectories or "strange attractors" that are expected to occur before we will be likely to say that the system (defined in a bounded region of $\mathbb{R}^{n}$ ) is "chaotic".

We stress the fact that distributional chaos is not uniform there. The most important property of the example presented is that it may be embedded in $\mathbb{R}^{3}$.

Example 8. Let us consider a discretization $f$ of a dynamical system defined on the phase space

$$
X=\left\{(x, y, z) \in \mathbb{R}^{3}: y^{2}+z^{2} \leq 1,|x| \leq z+1\right\}
$$

and with the phase portrait as in Fig. 1. We denote by $D$ the following set:

$$
D=\left\{\left(x, 0, \frac{1}{2}\right): x \in\left[-\frac{3}{2}, \frac{3}{2}\right]\right\} \text {. }
$$

The trajectory of $D$ is visualized as an interval which travels spirally from the center to the border of the cylinder containing $X$ (i.e. cylinder $y^{2}+z^{2} \leq 1$ ). This spiral motion guarantees that the diameter of iterations of $D$ changes cyclically from values close to 0 (phase 1) to values greater than the starting diameter (phase 2 ). The main idea of this example is to make time arrangement in a way that the described phases change with exponentially increasing delays, which in turn implies that any two distinct members of $D$ form a distributionally chaotic pair of type 1 
(in this case points from the set $\left\{(x, y, z): x^{2}+y^{2}=1\right.$ or $\left.y=z=0, x \in \mathbb{R}\right\} \cap X$ have to be fixed points of $f$ ).

Formal construction for Example 8. We start our construction by defining a map on the flat unit disc. Next it will be made three dimensional as presented in Fig. 1.

Step 1 (spiral curve). Let $d$ be the Euclidean metric in $\mathbb{R}^{2}$, and let $B \subset \mathbb{R}^{2}$ denote the unit disc. We define a spiral curve $F: \mathbb{R} \rightarrow B$ on the disk $B$ by the following formula:

$$
F(t)=\left(\left(\frac{1}{2}+\frac{1}{\pi} \arctan (t)\right) \sin (t),\left(\frac{1}{2}+\frac{1}{\pi} \arctan (t)\right) \cos (t)\right) .
$$

It can be easily verified that $\lim _{t \rightarrow \infty} d(F(t),(0,0))=1$ and $\lim _{t \rightarrow-\infty} d(F(t),(0,0))$ $=0$. The first step is to arrange a sequence $\left\{p_{j}\right\}_{j \in \mathbb{Z}} \subset F(\mathbb{R})$ and next to extend it to a map defined on the whole disc in a way that this sequence becomes the orbit of $p_{0}$.

Elements of the sequence with negative indices are irrelevant in our construction. The only thing we must control is that $\lim _{j \rightarrow \infty} p_{-j}=(0,0)$. It is enough to put $p_{j}=F\left(j \frac{\pi}{2}\right)$ for $j \leq 0$. We must pay more attention to points $p_{j}$ with indices $j>0$.

We start with two sequences which will allow us to make a proper arrangement of the number of iterations needed to delay transition between phases 1 and 2 . Let $m_{-1}=0$, let

$$
s_{0}=2, \quad v_{0}=\frac{\pi}{2}, \quad m_{0}=s_{0}
$$

and

$$
s_{n}=2^{2 n} m_{n-1}, \quad v_{n}=\frac{\pi}{s_{n}}, \quad m_{n}=m_{n-1}+s_{n} .
$$

Let $n \in \mathbb{N}$ and let $j$ be an integer such that $m_{n-1} \leq j<m_{n}$. We define:

$$
p_{j}=F\left(v_{n}\left(j-m_{n-1}\right)+n \pi\right) .
$$

Let us fix any $\varepsilon>0$. The map $F$ is continuous, so there exists an integer $K_{\varepsilon}>0$ such that for any $m>K_{\varepsilon}$ we have the following:

$$
\begin{aligned}
& d(y,(0,1))<\varepsilon \quad \text { for every } y \in F\left(\left[2 \pi m, 2 \pi m+\frac{\pi}{m}\right]\right), \\
& d(y,(0,-1))<\varepsilon \quad \text { for every } y \in F\left(\left[2 \pi m+\pi, 2 \pi m+\pi+\frac{\pi}{m}\right]\right) .
\end{aligned}
$$

This implies that for any $n>K_{\varepsilon}$ and integers which fulfill the inequalities

$$
m_{2 n-1} \leq i \leq\left(2^{n}+1\right) m_{2 n-1}, \quad m_{2 n} \leq j \leq\left(2^{n}+1\right) m_{2 n}
$$

the following conditions hold:

$$
d\left(p_{i},(0,1)\right)<\varepsilon, \quad d\left(p_{j},(0,-1)\right)<\frac{\varepsilon}{2} .
$$

In other words, the number of iterations during which the orbit of $p_{0}$ remains close to points $(0,-1)$ and $(0,1)$ exceeds the number of previous iterations with exponentially growing ratio.

Step 2 (disc). For any $\alpha \in[0,2 \pi)$ we define a map $F_{\alpha}: \mathbb{R} \rightarrow B$ by the formula:

$$
F_{\alpha}(t)=g_{\alpha}(F(t)),
$$

where

$$
g_{\alpha}(x, y)=(x \cos \alpha+y \sin \alpha,-x \sin \alpha+y \cos \alpha) .
$$




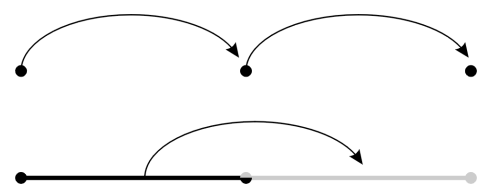

Figure 2. Idea of extension of $f$ from points to the whole curve

It is easily seen that each curve $F_{\alpha}(\mathbb{R})$ is obtained as the rotation of the curve $F(\mathbb{R})$ by the angle $\alpha$ around the point $(0,0)$. Obviously $F_{\alpha}(\mathbb{R}) \cap F_{\beta}(\mathbb{R})=\emptyset$ for $\alpha \neq \beta$ and $F=F_{0}$.

At the beginning we put $f\left(p_{n}\right)=p_{n+1}$. We may extend $f$ from points to $\operatorname{arcs}$ in a linear way (see Fig. 2) obtaining a continuous map $f: F(\mathbb{R}) \rightarrow F(\mathbb{R})$.

If we do the same on each curve $F_{\alpha}(\mathbb{R})$, then we obtain a continuous map $f$ : $E \rightarrow E$, where $E=B \backslash(\partial B \cup\{(0,0)\})$.

To finish our construction, we define $\left.f\right|_{(B \backslash E)}=i d_{(B \backslash E)}$. Note that the map $f$ : $B \rightarrow B$ is continuous and $f\left(p_{n}\right)=p_{n+1}$. This holds, because $\lim _{n \rightarrow+\infty} d\left(p_{n}, p_{n+1}\right)$ $=0$ and $\lim _{n \rightarrow-\infty} d\left(p_{n},(0,0)\right)=0$.

Step 3 (3d extension). Let $\rho$ denote the Euclidean metric in $\mathbb{R}^{3}$. We define a map $g:[-1,1] \times B \rightarrow[-1,1] \times B$ by putting $g(x, y, z)=(x, f(y, z))$. Let $\pi[-1,1] \times B \rightarrow$ $X$ denote the projection $\pi(x, y, z)=((z+1) x, y, z)$. Both functions are continuous, and additionally $\pi$ restricted to the set

$$
Q=\left\{(x, y, z): y^{2}+z^{2} \leq 1,|x| \leq z+1, z>-1\right\}
$$

is invertible, because for $(x, y, z) \in X \backslash\{(0,0,-1)\}$ we have the formula $\pi^{-1}(x, y, z)$ $=\left(\frac{x}{z+1}, y, z\right) \in Q$ (in fact, $\pi^{-1}: \pi(Q) \rightarrow Q$ is a homeomorphism).

The factor map $\tilde{f}: X \rightarrow X$ given by

$$
\widetilde{f}(x, y, z)= \begin{cases}\pi\left(g\left(\pi^{-1}(x, y, z)\right)\right), & z \neq-1 \\ (0,0,-1), & z=-1\end{cases}
$$

is well defined and continuous. The dynamics of the map $\tilde{f}$ is very regular. Every point from the set $Y=\pi([-1,1] \times(\partial B \cup\{(0,0)\}))$ is a stationary point and each point $(x, y, z) \in X \backslash Y$ behaves exactly the same way as $(x, y)$ on the disc $B$. Strictly speaking, it is repelled by the center of the cylinder $y^{2}+z^{2}=1$ (i.e. by the set $[-1,1] \times\{0\} \times\{0\})$ and tends asymptotically to the limit circle $\pi([-1,1] \times \partial B)$ consisting of stationary points. Denote $p_{j}=\left(p_{j}^{(1)}, p_{j}^{(2)}\right)$ for $j \in \mathbb{Z}$. Then

$$
\begin{aligned}
\tilde{f}\left(x, p_{j}^{(1)}, p_{j}^{(2)}\right) & =\pi\left(g\left(\pi^{-1}\left(x, p_{j}^{(1)}, p_{j}^{(2)}\right)\right)\right)=\pi\left(g\left(\frac{x}{p_{j}^{(2)}+1}, p_{j}^{(1)}, p_{j}^{(2)}\right)\right) \\
& =\pi\left(\frac{x}{p_{j}^{(2)}+1}, f\left(p_{j}\right)\right)=\pi\left(\frac{x}{p_{j}^{(2)}+1}, p_{j+1}^{(1)}, p_{j+1}^{(2)}\right) \\
& =\left(\frac{p_{j+1}^{(2)}+1}{p_{j}^{(2)}+1} x, p_{j+1}^{(1)}, p_{j+1}^{(2)}\right)
\end{aligned}
$$


and as a direct consequence of these calculations,

$$
\tilde{f}^{j}\left(x, p_{0}^{(1)}, p_{0}^{(2)}\right)=\left(\frac{p_{j}^{(2)}+1}{p_{0}^{(2)}+1} x, p_{j}^{(1)}, p_{j}^{(2)}\right)=\left(\frac{2}{3}\left(p_{j}^{(2)}+1\right) x, p_{j}^{(1)}, p_{j}^{(2)}\right) .
$$

Step 4 (scrambled set). Let us consider the following set:

$$
D=\left\{\left(x, p_{0}^{(1)}, p_{0}^{(2)}\right),|x| \leq p_{0}^{(2)}+1\right\}=\left\{\left(x, 0, \frac{1}{2}\right),|x| \leq \frac{3}{2}\right\} .
$$

We claim that $D$ is a distributionally scrambled set (of type 1 ).

Fix any distinct $\alpha, \beta \in\left[-\frac{3}{2}, \frac{3}{2}\right]$ and let $z^{\gamma}$ denote the point $z^{\gamma}=\left(\gamma, 0, \frac{1}{2}\right) \in D$ provided that $\gamma \in\left[-\frac{3}{2}, \frac{3}{2}\right]$. It directly follows from the time parametrization we have made that for any $\varepsilon>0, n>K_{\varepsilon}$ and $m_{2 n} \leq i \leq\left(2^{n}+1\right) m_{2 n}$ the following inequality holds:

$$
\begin{aligned}
\rho\left(\widetilde{f}^{i}\left(z^{\alpha}\right), \tilde{f}^{i}\left(z^{\beta}\right)\right) & =\left|\frac{2}{3}\left(p_{i}^{(2)}+1\right) \alpha-\frac{2}{3}\left(p_{i}^{(2)}+1\right) \beta\right| \leq 2\left|p_{i}^{(2)}+1\right| \\
& \leq 2 d\left(p_{i},(0,-1)\right)<\varepsilon .
\end{aligned}
$$

Then for $n$ large enough we obtain an upper bound:

$$
\frac{1}{\left(2^{n}+1\right) m_{2 n}} \# \xi\left(z^{\alpha}, z^{\beta}, \varepsilon,\left(2^{n}+1\right) m_{2 n}\right) \geq \frac{2^{n} m_{2 n}}{\left(2^{n}+1\right) m_{2 n}} \longrightarrow 1 .
$$

By the same arguments, if $n>K_{\frac{1}{8}}$ and $m_{2 n-1} \leq i \leq\left(2^{n}+1\right) m_{2 n-1}$, then

$$
\begin{aligned}
\rho\left(\widetilde{f}^{i}\left(z^{\alpha}\right), \widetilde{f}^{i}\left(z^{\beta}\right)\right) & \geq \frac{2}{3}\left(p_{i}^{(2)}+1\right)|\alpha-\beta| \geq \frac{2}{3}\left(2-\left|p_{i}^{(2)}-1\right|\right)|\alpha-\beta| \\
& \geq \frac{2}{3}\left(2-d\left(p_{i},(0,1)\right)\right)|\alpha-\beta| \geq \frac{4-\frac{2}{8}}{3}|\alpha-\beta| \\
& >|\alpha-\beta|,
\end{aligned}
$$

which implies that

$$
\frac{1}{\left(2^{n}+1\right) m_{2 n-1}} \# \xi\left(z^{\alpha}, z^{\beta},|\alpha-\beta|,\left(2^{n}+1\right) m_{2 n-1}\right) \leq \frac{m_{2 n-1}}{\left(2^{n}+1\right) m_{2 n-1}} \longrightarrow 0 .
$$

We have just obtained that $F_{z^{\alpha} z^{\beta}}^{*}(|\alpha-\beta|)=0$ and $F_{z^{\alpha} z^{\beta}}^{*}(\varepsilon)=1$ for any $\varepsilon>0$.

\section{Strengthened Definitions}

In the previous section we observed that if the distributional chaos (in the strongest sense $D C 1$ ) is not assumed to be uniform, then it is possible to construct a "regular" dynamical system embedded in $\mathbb{R}^{3}$ (which may even be visualized as a discretization of a continuous flow on the unit ball) which fulfills this definition.

Then a natural approach is to consider uniform distributional chaos. Unfortunately, it is possible to construct a space $X$ and a map $f: X \rightarrow X$ such that $f$ has only two fixed points and all other points oscillate between them; however, this is a rather philosophical problem, if this system has regular trajectories, as $X$ is a Cantor set. The example similar to that presented below was first discovered by R. Pikuła in [47. As a general method for such examples, a construction was independently obtained in [46. 
Example 9. We will construct a (one-sided) symbolic system over a two-letter alphabet (0 and 1). Let us consider sequences $s_{0}=1, m_{n}=\sum_{i=1}^{n} s_{n}$ and $s_{n+1}=$ $2^{n+1} m_{n}$. We define words $u(0, n)=0^{s_{n}}, u(1, n)=1^{s_{n}}$ and a set $X \subset \Sigma^{\omega}$ as

$$
X=\operatorname{cl}\left(\bigcup_{n \in \mathbb{N}} \sigma^{n}\left(\left\{u\left(x_{0}, 0\right) u\left(x_{1}, 1\right) u\left(x_{2}, 2\right) \ldots: x \in \Sigma^{\omega}\right\}\right)\right),
$$

where $\operatorname{cl}(A)$ denotes the closure of set $A$. It is easy to verify that $X$ is compact and $\sigma(X) \subset X$. Observe that $h_{\text {top }}\left(\left.\sigma\right|_{Y}\right)=0$ and $\Omega\left(\left.\sigma\right|_{X}\right)=Y$, provided

$$
Y=\left\{0^{\infty}, 1^{\infty}\right\} \cup \bigcup_{k \in \mathbb{N}}\left\{0^{k} 1^{\infty}, 1^{k} 0^{\infty}\right\}
$$

It is caused by the fact that any element of $x \in X$ may contain at most one block of the form $10^{k} 1$ or $01^{k} 0$, where $k$ is a fixed integer (this observation is an immediate consequence of the inequality $\left.s_{n+1}>s_{1}+\ldots+s_{n}\right)$. This implies that $h_{t o p}\left(\left.\sigma\right|_{X}\right)=0$ (see [13]). Additionally $X$ contains an uncountable distributionally chaotic set of type 1 and the distributional chaos is uniform.

Construction of a distributionally scrambled set. Let us define a parameters set:

$$
\Gamma=\left\{x_{0} 0 x_{0} x_{1} 0 x_{0} x_{1} x_{2} 0 x_{0} \ldots: x \in \Sigma^{\omega}\right\} .
$$

We claim that the set

$$
D=\left\{x^{(\alpha)}=u\left(\alpha_{0}, 0\right) u\left(\alpha_{1}, 1\right) u\left(\alpha_{2}, 2\right) \ldots: \alpha \in \Gamma\right\}
$$

is a distributionally scrambled set of type 1 . Let us take any distinct $\alpha, \beta \in \Gamma$. There exists a sequence $\left\{\nu_{j}\right\}$ such that $\alpha_{\nu_{j}}=\beta_{\nu_{j}}=0$ for all $j \in \mathbb{N}$. Then

$$
\begin{aligned}
\frac{1}{m_{\nu_{j}}} \xi\left(x^{(\alpha)}, x^{(\beta)}, \frac{1}{2^{k}}, m_{\nu_{j}}\right) & =\frac{1}{m_{\nu_{j}}} \#\left\{i: x_{[i, i+k)}=y_{[i, i+k)}, i<m_{\nu_{j}}\right\} \\
& \geq \frac{\left|u\left(0, \nu_{j}\right)\right|-2 k}{m_{\nu_{j}}} \geq \frac{s_{\nu_{j}}-2 k}{m_{\nu_{j}}} \\
& \geq \frac{2^{\nu_{j}} m_{\nu_{j}-1}-2 k}{\left(2^{\nu_{j}}+1\right) m_{\nu_{j}-1}} \longrightarrow 1 .
\end{aligned}
$$

By the construction of $\Gamma$, there also exists a sequence $\left\{\mu_{j}\right\}$ such that $\alpha_{\mu_{j}} \neq \beta_{\mu_{j}}$. We obtain that

$$
\begin{aligned}
\frac{1}{m_{\mu_{j}}} \xi\left(x^{(\alpha)}, x^{(\beta)}, \frac{2}{3}, m_{\mu_{j}}\right) & =\frac{1}{m_{\nu_{j}}} \#\left\{i: x_{i}=y_{i}, i<m_{\mu_{j}}\right\} \\
& \leq \frac{\left|u\left(0, \mu_{j}\right)\right|}{m_{\nu_{j}}} \leq \frac{m_{\mu_{j}-1}}{\left(2^{\mu_{j}}+1\right) m_{\mu_{j}-1}} \longrightarrow 0,
\end{aligned}
$$

which ends the proof.

By the definition, $\left.\sigma\right|_{X}$ is not transitive. Additionally, if we extend $X$ by including one isolated periodic orbit, e.g.

$$
M=X \cup\left\{(01)^{\infty},(10)^{\infty}\right\},
$$

then it is also clear that there is no dense distributional scrambled set in $M$.

The above-mentioned situation does not change if we restrict our considerations to the case of surjective or even invertible dynamical systems. Namely, it is enough to define the following set (now, $\sigma$ operates on bi-infinite sequences):

$$
X=\operatorname{cl}\left(\bigcup_{n \in \mathbb{Z}} \sigma^{n}\left(\left\{\ldots 00 \dot{0} u\left(x_{0}, 0\right) u\left(x_{1}, 1\right) u\left(x_{2}, 2\right) \ldots: x \in \Sigma^{\omega}\right\}\right)\right) \subset{ }^{\omega} \Sigma^{\omega} .
$$


Again, $h_{\text {top }}\left(\left.\sigma\right|_{X}\right)=0,\left.\sigma\right|_{X}$ exhibits uniform distributional chaos and as before $\left.\sigma\right|_{X}$ is not transitive. However, this time $\left.\sigma\right|_{X}$ is a homeomorphism.

This motivates us to state the following definition.

Definition 10. Let $f: X \rightarrow X$ be a continuous map acting on a compact metric space $(X, d)$. Suppose that $D$ is an uncountable distributionally scrambled set of type 1 for $f$ and the distributional chaos is uniform.

We say that $f$ exhibits dense distributional chaos if the set $D$ may be chosen to be dense. If $D$ is not only dense but additionally consists of points with dense orbits, then we say that $f$ exhibits transitive distributional chaos.

Transitive distributional chaos is present in many dynamical systems considered as being chaotic. It was proved in 45] that the specification property (as introduced by Bowen in 13) implies this kind of chaos (the study of relations between the specification property and distributional chaos originated from [53]). This implies that mixing shifts of finite type or an even wider class of nontrivial mixing hyperbolic homeomorphisms exhibit transitive distributional chaos. As we mentioned earlier, dense distributional chaos implies dense $\varepsilon$-chaos, which implies sensitivity. Additionally, it is easily seen that in the case of transitive distributional chaos, the set $X$ may not contain isolated points and as a result $f$ is transitive (see the proof of Theorem 11). Then, transitive distributional chaos is a notion stronger than those proposed by Wiggins or Martelli. Before we give the formal proof, let us first recall these definitions.

Let $f$ be a continuous map from a compact metric space $(X, d)$ into itself. The orbit of a point $x \in X$ is said to be unstable if there exists $r>0$ such that for every $\varepsilon>0$ there are $y \in X$ and $n \geq 1$ satisfying the inequalities $d(x, y)<\varepsilon$ and $d\left(f^{n}(x), f^{n}(y)\right)>r$. The map $f$ is said to be chaotic in the sense of Martelli (see [38]) if there exists $x_{0} \in X$ such that $x_{0}$ has a dense orbit which is unstable.

Theorem 11. If a dynamical system $(X, f)$ exhibits transitive distributional chaos, then it is chaotic in the sense of Martelli.

Proof. There are no isolated points in $X$ as otherwise the set of points with dense orbit would be at most countable. But in the case of compact set without isolated points, the existence of a dense orbit implies transitivity.

Let $D$ be a dense scrambled set consisting of transitive points and let $r>0$ be such that $F_{x y}(r)=0$ for all distinct $x, y \in D$. Let us fix any $x_{0} \in D$. Then the orbit of $x_{0}$ is dense and arbitrarily close to $x_{0}$ and we may find some other point $y \in D$. The condition $F_{x_{0} y}(r)=0$ implies that $d\left(f^{n}(x), f^{n}(y)\right)>r$ for some $n$.

According to Wiggins 61, a continuous map $f: X \rightarrow X$ is chaotic if it is transitive and has sensitive dependence on initial conditions. This kind of chaos is also known under the name Auslander-Yorke chaos [4.

Theorem 12. If a dynamical system $(X, f)$ exhibits transitive distributional chaos, then it is chaotic in the sense of Wiggins.

Proof. Fix any $\varepsilon>0$. In an $\varepsilon$-neighborhood of any point $x$ we can find two points $y, z \in D$ such that $d\left(f^{n}(y), f^{n}(z)\right)>r$. But then $d\left(f^{n}(x), f^{n}(y)\right)>\frac{r}{2}$ or $d\left(f^{n}(x)\right.$, $\left.f^{n}(z)\right)>\frac{r}{2}$.

Theorem 13. If $(X, f),(Y, g)$ are topologically conjugate dynamical systems acting on compact metric spaces, then $f$ exhibits dense (or transitive) distributional chaos if so does $g$. 
Proof. It was proved in [58 that if $\phi: X \rightarrow Y$ is topological conjugacy between $f$ and $g$ (i.e. $\phi$ is a homeomorphism and $\phi \circ f=g \circ \phi$ ) and $D$ is a distributionally scrambled set for $f$, then $\phi(D)$ is a distributionally scrambled set for $g$. The remaining part of the proof is straightforward (density of sets transfers through homeomorphisms).

\section{Proximal Relation and distributional chaos}

The notion of proximality is an essential part of the definition of distributional chaos ( $D C 1$ and $D C 2$ ). In this section we will recall some basic facts on the proximal relation with emphasis on their applications to the theory of distributional chaos. More deep treatment of this topic (abstract topological flows on not necessarily compact spaces) may be found in [26] and [3].

Let $(X, \rho)$ be a compact metric space and $f: X \rightarrow X$ be continuous. A pair of points $(x, y) \in X \times X$ is proximal if $\liminf _{n \rightarrow \infty} \rho\left(f^{n}(x), f^{n}(y)\right)=0$ and distal if it is not proximal. A set $A \subset \mathbb{N}$ is syndetic if there exists a positive integer $a$ such that

$$
\{i, i+1, \ldots, i+a\} \cap A \neq \emptyset
$$

for any $i \in \mathbb{N}$. A pair of points $(x, y) \in X \times X$ is syndetically proximal if for every $\varepsilon>0$ the following set,

$$
A_{x y}^{\varepsilon}=\left\{j \in \mathbb{N}: \rho\left(f^{j}(x), f^{j}(y)\right)<\varepsilon\right\},
$$

is syndetic.

We define the proximal relation and the syndetically proximal relation on $X \times X$ by the formulae:

$$
\begin{aligned}
& \mathrm{P}(f)=\{(x, y) \in X \times X:(x, y) \text { is proximal }\}, \\
& \mathrm{L}(f)=\{(x, y) \in X \times X:(x, y) \text { is syndetically proximal }\} .
\end{aligned}
$$

It is easy to see that both relations are symmetric, reflexive and $\mathrm{L}(f) \subset \mathrm{P}(f)$. For any relation $R \subset X \times X$ and any point $x \in X$, we write

$$
R(x)=\{y:(x, y) \in R\} .
$$

The set $\mathrm{P}(f)(x)$ is called the proximal cell of $x$.

We say that $f$ is proximal if $\mathrm{P}(f)=X \times X$. A map $f$ is a proximal extension of a map $g: Y \rightarrow Y$ (by a factor map $\phi: X \rightarrow Y$ ) if whenever $x, y \in X$ with $\phi(x)=\phi(y)$, then $x$ and $y$ are proximal.

The following fact is very important from the point of view of distributional chaos.

Theorem 14. If $f$ is acting on a compact metric space $(X, \rho)$ and $\mathrm{P}(f)$ is closed, then $\mathrm{L}(f)=\mathrm{P}(f)$. In particular, if $f$ is proximal, then $\mathrm{L}(f)=X \times X$.

Proof. When $f$ is a homeomorphism, the proof is a special case of [17, Thm. 3]. The proof for the noninvertible case follows the same lines.

Corollary 15. If $f$ acting on a compact metric space $(X, \rho)$ is proximal, then there is no DC1 pair in $X$.

Proof. The proof follows directly from the definition of a syndetically proximal pair (and Theorem 14). Let us fix any pair of distinct points $x, y \in X$ and let $\varepsilon>0$. 
There exists a positive integer $M_{\varepsilon}$ such that for every $i \in \mathbb{N}$ the following condition holds:

$$
\left\{i, i+1, \ldots, i+M_{\varepsilon}\right\} \cap A_{x y}^{\varepsilon} \neq \emptyset .
$$

Observe that $M_{\varepsilon}\left\lfloor\frac{n}{M_{\varepsilon}}\right\rfloor \leq n \leq\left(M_{\varepsilon}+1\right)\left\lfloor\frac{n}{M_{\varepsilon}}\right\rfloor$ for any $n \in \mathbb{N}$, where $\lfloor r\rfloor=a$ is the greatest integer such that $a \leq r$. Observe that

$$
\frac{1}{n} \xi(x, y, \varepsilon, n) \geq \frac{1}{n}\left\lfloor\frac{n}{M_{\varepsilon}}\right\rfloor \geq \frac{1}{M_{\varepsilon}+1} .
$$

This implies that $F_{x y}(\varepsilon)>0$ for any $\varepsilon>0$.

Remark 16. Note that Corollary 15] does not exclude the possibility that there are $D C 2$ pairs for $f$. In Section 7 we will present an example of a proximal system which exhibits distributional chaos of type 2 .

Lemma 17. If $f: X \rightarrow X$ is a proximal extension of a minimal system $g: Y \rightarrow Y$ (i.e. $Y$ is minimal for $g$ ), then $X$ contains a unique minimal set (for $f$ ).

Proof. The proof is easy and may be found in [26].

Lemma 18. A continuous map $f: X \rightarrow X$ is proximal iff it has a fixed point which is the unique minimal subset of $X$.

Proof. It is easy to see that if $f$ is proximal, then it has a fixed point, so applying Lemma 17 we obtain the first implication ( $f$ is a proximal extension of the onepoint system). Conversely, if $f$ has the fixed point $p$ which is the unique minimal subset of $X$, then $(p, p)$ has the same property for $f \times f$. But it is a known fact that $f$ is proximal iff all minimal sets of $f \times f$ are contained in the diagonal. (25] contains the proof for abstract flows, in particular homeomorphisms. The proof for the noninvertible case is identical.) Another proof of this lemma may be found in [1. Prop. 2.2].

Remark 19. Although Lemma 18 is simple, it provides a very nice tool to decide whether a given system is proximal or not. We will use it in a further part of the article.

Theorem 20. For a minimal dynamical system $(X, f)$ the following conditions are equivalent:

(1) The system is weak mixing.

(2) For every $x \in X$, the proximal cell $\mathrm{P}(f)(x)$ is dense in $X$.

(3) For some $x \in X$, the proximal cell $\mathrm{P}(f)(x)$ is dense in $X$.

(4) $\mathrm{P}(f)$ is dense in $X \times X$.

Proof. The same theorem is valid for general flows (group actions) [3, Ch. 9, Thm. 13]. However there is an additional assumption that the flow admits an invariant Borel probabilistic measure. By the Krylov-Bogolubov theorem $f$ always admits an invariant Borel probabilistic measure; thus the case of $f$ being a homeomorphism is covered by [3]. The proof for arbitrary continuous maps acting on compact metric spaces is given in [1, Prop. 3.10].

Corollary 21. If a minimal dynamical system $(X, f)$ exhibits dense $\varepsilon$-chaos, then $f$ is weakly mixing. 
Proof. This is an immediate consequence of Theorem 20. Namely, if $D$ is a dense scrambled set, then the proximal cell of any point $x \in D$ contains $D$, in particular is dense.

When restricted to the case of minimal systems, dense distributional chaos and transitive distributional chaos are equivalent notions (transitive distributional chaos always implies dense distributional chaos; in the case of a minimal system, the orbit of each point is dense, which gives the converse implication). Dense $\varepsilon$-chaos is implied by dense distributional chaos, so every dense distributionally chaotic minimal system is weakly mixing.

\section{UNCOUNTABLE FAMILY OF MINIMAL SYSTEMS}

The idea of the construction of a minimal distributionally chaotic system was first presented in [35. Further properties of this system were discussed in 15. We generalize this construction to obtain an uncountable family of minimal sets with some special properties (in particular, dynamics on each of these sets displays uniform distributional chaos). Each step of the construction is also slightly different when compared to [35, but it makes the calculations easier. The main tool in our construction is the recurrence index; however, we do not use Sturmian sequences (the main tool used in 42 to obtain an uncountable family of minimal sets in $\Sigma^{\omega}$ ) because Sturmian systems are not weakly mixing.

Let $r=\left(r_{1}, r_{2}, \ldots\right) \subset \mathbb{N}^{\mathbb{N}}$ be any sequence of positive integers. Let us define words $A_{1}=1, B_{1}=0$ and perform an inductive construction. Suppose that for some $n \in \mathbb{N}$, the words $A_{1}, B_{1}, \ldots, A_{n}, B_{n}$ are defined. Let $J_{i}^{(0)}=A_{i}^{r_{i}}, J_{i}^{(1)}=B_{i}^{r_{i}}$. We define a set $P_{n} \subset \Sigma^{*}$ by

$$
P_{n}=\left\{J_{1}^{\left(a_{1}\right)} J_{2}^{\left(a_{2}\right)} \ldots J_{n}^{\left(a_{n}\right)}: a \in \Sigma^{n}\right\} .
$$

We enumerate elements of $P_{n}$, let say $P_{n}=\left\{w_{1}^{n}, \ldots, w_{2^{n}}^{n}\right\}$ and let $A_{n+1}$ be a catenation of all possible pairs formed by elements of $P_{n}$, i.e.

$$
A_{n+1}=w_{1}^{n} w_{1}^{n} w_{2}^{n} w_{1}^{n} \ldots w_{2^{n}-1}^{n} w_{2^{n}}^{n} w_{2^{n}}^{n} w_{2^{n}}^{n} .
$$

Denote $B_{n+1}=\overline{A_{n+1}}$. We stress the fact that the words $A_{n}, B_{n}$ may be different for different sequences $r \in \mathbb{N}^{\mathbb{N}}$.

Remark 22. For any positive integers $m, n \in \mathbb{N}, n \leq m$ there exist a positive integer $k$ and a finite sequence of indices $i_{1}, \ldots, i_{k} \in\left\{1, \ldots, 2^{n}\right\}$ such that

$$
A_{m}=w_{i_{1}}^{n} w_{i_{2}}^{n} \ldots w_{i_{k}}^{n} .
$$

Let us define $x^{(r)}=A_{1}^{r_{1}} A_{2}^{r_{2}} A_{3}^{r_{3}} \ldots$ and $M^{(r)}=\omega\left(x^{(r)}, \sigma\right)$. Given $n \in \mathbb{N}$ we will denote $s_{n}^{(r)}=\left|A_{n}\right|$.

Theorem 23. For any $n \in \mathbb{N}$ the following inequalities hold:

$$
r_{n} s_{n}^{(r)} \leq R\left(s_{n}^{(r)}+1, x\right) \leq 32^{n} r_{n} r_{n+1} s_{n}^{(r)} .
$$

Proof. Observe that $A_{1}^{r_{1}} A_{2}^{r_{2}} \ldots A_{n}^{r_{n}}=J_{1}^{(0)} J_{2}^{(0)} \ldots J_{n}^{(0)}$. This combined with (22.1) implies that $x^{(r)}$ is a concatenation of words from $P_{n}$. But then any subword of $x$ with length $s_{n}^{(r)}+1$ is a subword of a word $u w$, where $u, w \in P_{n}$, so it is a subword 
of $A_{n+1}$ and obviously it is also a subword of $B_{n+1}$. But any element of $P_{n+1}$ contains $A_{n+1}$ or $B_{n+1}$. Let us fix any $v \in P_{n+1}$. Then

$$
\begin{aligned}
|v| & =\left|J_{1}^{(0)} J_{2}^{(0)} \ldots J_{n+1}^{(0)}\right|=\left|J_{1}^{(0)} J_{2}^{(0)} \ldots J_{n}^{(0)}\right|+\left|J_{n+1}^{(0)}\right| \leq\left|A_{n+1}\right|+r_{n+1}\left|A_{n+1}\right| \\
& \leq 2 r_{n+1} s_{n+1}^{(r)}
\end{aligned}
$$

and additionally

$$
\begin{aligned}
\left|A_{n+1}\right| & =\sum_{u \in P_{n}} \sum_{w \in P_{n}}|u w|=\sum_{u \in P_{n}} \sum_{w \in P_{n}} 2|w| \leq 2\left(2^{n}\right)^{2} 2 r_{n} s_{n}^{(r)} \\
& \leq 2^{2 n+2} r_{n} s_{n}^{(r)} .
\end{aligned}
$$

Finally, we obtain that

$$
|v| \leq 2 r_{n+1} 2^{2 n+2} r_{n} s_{n}^{(r)} \leq 32^{n} r_{n+1} r_{n} s_{n}^{(r)} .
$$

We have just proved that

$$
R\left(s_{n}^{(r)}+1, x\right) \leq 32^{n} r_{n} r_{n+1} s_{n}^{(r)} .
$$

The lower bound is an immediate consequence of Theorem 7 ,

Corollary 24. Point $x^{(r)}$ (or equivalently $M^{(r)}$ ) is minimal for any sequence $r \in$ $\mathbb{N}^{\mathbb{N}}$.

Theorem 25. A dynamical system $\left(M^{(r)},\left.\sigma\right|_{M^{(r)}}\right)$ has zero topological entropy.

Proof. Every point $x \in M^{(r)}$ is a concatenation of words from $P_{n}$. All words form $P_{n}$ have the same length $\nu_{n}=|w|$ provided $w \in P_{n}$. Any subword of $x$ is a subword of $u w$ for some $u, w \in P_{n}$. This implies that

$$
\# B_{\nu_{n}}\left(M^{(r)}\right) \leq \nu_{n}\left(\# P_{n}\right)^{2} \leq \nu_{n}\left(2^{n}\right)^{2}=\nu_{n} 4^{n}
$$

and as a result,

$$
h_{t o p}\left(\left.\sigma\right|_{M^{(r)}}\right)=\lim _{n \rightarrow+\infty} \frac{\log \# B_{\nu_{n}}\left(M^{(r)}\right)}{\nu_{n}} \leq \lim _{n \rightarrow+\infty}\left(\frac{\log \nu_{n}}{\nu_{n}}+\frac{n \log 4}{\nu_{n}}\right)=0 .
$$

Theorem 26. A dynamical system $\left(M^{(r)},\left.\sigma\right|_{M^{(r)}}\right)$ exhibits uniform distributional chaos (i.e. it has an uncountable scrambled set of type 1 which consists of transitive points and the chaos is uniform).

Proof. Similarly to previous constructions, we have to start with a parameters set. For any $\alpha \in(0,1)$ let $\hat{\alpha}=\left(\alpha_{0}, \alpha_{1}, \ldots\right)$ be one of its at most two binary representations (finite or infinite), i.e.

$$
\alpha=\sum_{i=0}^{\infty} \frac{1}{2^{i+1}} \alpha_{i} .
$$

We use $\hat{\alpha}$ to define another sequence $\tilde{\alpha}$ given by:

$$
\tilde{\alpha}=\left(\alpha_{0}, 0, \alpha_{0}, \alpha_{1}, 0, \alpha_{0}, \alpha_{1}, \alpha_{2}, 0, \alpha_{0}, \ldots\right) .
$$

Let $N$ be the maximal integer such that $\alpha<\frac{1}{N}$. The last modification of the sequence $\hat{\alpha}$ is $\alpha^{\prime}$ is defined by the following formula:

$$
\alpha_{i}^{\prime}= \begin{cases}0, & i \leq N \\ \tilde{\alpha}_{i}, & i>N .\end{cases}
$$


It directly follows from the definition that for any $\alpha, \beta \in(0,1), \alpha \neq \beta$ there exist increasing sequences $\nu_{i}, \mu_{i}$ such that $\alpha_{\nu_{i}}^{\prime}=\beta_{\nu_{i}}^{\prime}$ and $\alpha_{\mu_{i}}^{\prime} \neq \beta_{\mu_{i}}^{\prime}$. Let $\Gamma=$ $\left\{\alpha^{\prime}: \alpha \in(0,1)\right\}$.

For any $\gamma \in \Gamma$ we define

$$
z(\gamma)=J_{1}^{\left(\gamma_{1}\right)} J_{2}^{\left(\gamma_{2}\right)} J_{3}^{\left(\gamma_{3}\right)} \ldots
$$

It follows from the definition that $\left|J_{n}^{(0)}\right|=\left|J_{n}^{(1)}\right|$ and

$$
\left|J_{n+1}^{(0)}\right| \geq 2^{n}\left|J_{1}^{(0)} \ldots J_{n}^{(0)}\right|=2^{n} \sum_{i=1}^{n}\left|J_{i}^{(0)}\right| .
$$

By this observation, the proof that $D=\{z(\gamma): \gamma \in \Gamma\}$ is a distributionally scrambled set of type 1 (for the dynamical system $\left(\Sigma^{\omega}, \sigma\right)$ ) and distributional chaos is uniform is exactly the same as the proof performed for Example 9

It remains to show that $D \subset M^{(r)}$. It follows from the definition that for any $n>0$ there exists $m>n$ such that the word $J_{1}^{\left(\gamma_{1}\right)} J_{2}^{\left(\gamma_{2}\right)} \ldots J_{n}^{\left(\gamma_{n}\right)}$ is a subword of $A_{m}$. This implies that $D \subset M^{(r)}$.

Theorem 27. There exists an uncountable set $\Xi \subset \mathbb{N}^{\mathbb{N}}$ such that for any distinct $\nu, \mu \in \Xi$, sets $M^{(\nu)}$ and $M^{(\mu)}$ are distinct (have empty intersection).

Proof. We define a function $r: \Sigma^{\omega} \rightarrow \mathbb{N}^{\mathbb{N}}$ by the formula:

$$
r(\alpha)_{i}= \begin{cases}64^{\alpha_{k} i}, & i=2 k \\ 1, & i=2 k+1 .\end{cases}
$$

We claim that $\Xi=\left\{r(\alpha): \alpha \in \Sigma^{\omega}\right\}$ is the desired parameters set (obviously it is uncountable).

Let us take any distinct $\mu, \nu \in \Xi$ and let $n \geq 0$ be the first position such that $\mu_{n} \neq \nu_{n}$. It follows from the definition of the function $r$ that $n=2 k$ for some $k \in \mathbb{N}$. Furthermore, observe that $s_{n}^{(\mu)}=s_{n}^{(\nu)}$ because $\mu_{i}=\nu_{i}$ for $i=1, \ldots, n-1$ and these numbers define $A_{n}$ uniquely. We may also assume that $\mu_{n}=64^{n}$ and $\nu_{n}=\nu_{n+1}=\mu_{n+1}=1$. Let us denote $K=s_{n}^{(\mu)}=s_{n}^{(\nu)}$. It immediately follows that

$$
R\left(K+1, x^{(\nu)}\right) \leq 32^{n} \nu_{n} \nu_{n+1} K<64^{n} K=\mu_{n} K \leq R\left(K+1, x^{(\mu)}\right)
$$

and by Theorem 6 the proof is finished.

\section{PikUŁa's EXAMPle}

Recently, R. Pikuła (motivated by [24, Ex. 3.4]) constructed in [47] a subshift which has positive topological entropy but no $D C 1$ pair. We present this construction here (the construction is slightly different but the main idea is exactly the same). In addition we prove that the constructed system (in fact a family of systems) has an uncountable distributionally scrambled set of type 2 . The main idea here is to force the regular presence of longer and longer blocks of zeros in each point of the constructed subshift. In other words, we will construct a proximal dynamical system with some additional properties.

Let $s<t$ be any two positive integers and let $\mathcal{A}=\{0, \diamond\}$. We define an infinite periodic word $p^{(s, t)}$ over $\mathcal{A}$ by

$$
p^{(s, t)}=\left(\diamond^{t-s} 0^{s}\right)^{\infty}
$$


Observe that for any integer $n>0$ the following inequality holds:

$$
\frac{1}{n}\left|p_{[0, n)}^{(s, t)}\right|_{0} \leq \frac{s}{t}
$$

For any positive integer $k$ we define a set $Q^{(s, t, k)}$ by

$$
Q^{(s, t, k)}=\left\{x \in \Sigma_{k}^{\omega}: \text { if } p_{i}^{(s, t)}=0, \text { then } x_{i}=0\right\} \subset \Sigma_{k}^{\omega},
$$

where $\Sigma_{k}=\{0, \ldots, k-1\}$.

Definition 28. Let $k$ be a positive integer and let $\hat{s}=\left\{s_{i}\right\}_{i \in \mathbb{N}}, \hat{t}=\left\{t_{i}\right\}_{i \in \mathbb{N}}$ be increasing sequences of positive integers such that $\sum_{i=1}^{\infty} \frac{s_{i}}{t_{i}}<1$. We define a set $\mathcal{C}^{(\hat{s}, \hat{t}, k)}$ by the formula:

$$
\mathcal{C}^{(\hat{s}, \hat{t}, k)}=\bigcap_{i=1}^{\infty} \bigcup_{j=1}^{\infty} \sigma^{j}\left(Q^{\left(s_{i}, t_{i}, k\right)}\right) .
$$

Theorem 29. Let $k$ be a positive integer and let $\hat{s}=\left\{s_{i}\right\}_{i \in \mathbb{N}}, \hat{t}=\left\{t_{i}\right\}_{i \in \mathbb{N}}$ be increasing sequences of positive integers such that $\sum_{i=1}^{\infty} \frac{s_{i}}{t_{i}}<1$. In this case, the set $\mathcal{C}^{(\hat{s}, \hat{t}, k)}$ is closed and a $\sigma$-invariant subset of $\Sigma_{k}^{\omega}$.

Proof. Observe that $Q^{\left(s_{i}, t_{i}, k\right)}$ is closed and

$$
\bigcup_{j=1}^{\infty} \sigma^{j}\left(Q^{\left(s_{i}, t_{i}, k\right)}\right)=\bigcup_{j=1}^{t_{i}} \sigma^{j}\left(Q^{\left(s_{i}, t_{i}, k\right)}\right) .
$$

Then $\mathcal{C}^{(\hat{s}, \hat{t}, k)}$ is closed as it is an intersection of closed sets and it is invariant by the definition.

Theorem 30. Let $k$ be a positive integer and let $\hat{s}=\left\{s_{i}\right\}_{i \in \mathbb{N}}, \hat{t}=\left\{t_{i}\right\}_{i \in \mathbb{N}}$ be increasing sequences of positive integers such that $\sum_{i=1}^{\infty} \frac{s_{i}}{t_{i}}<1$. In this case,

$$
h_{\text {top }}\left(\left.\sigma\right|_{\mathcal{C}^{(\hat{s}, \hat{t}, k)}}\right) \geq \theta \log k,
$$

where $\theta=1-\sum_{i=1}^{\infty} \frac{s_{i}}{t_{i}}$.

Proof. Let us consider an infinite word $z$ over $\{0, \diamond\}$ such that

$$
z_{j}=\diamond \quad \Longleftrightarrow \quad p_{j}^{\left(s_{i}, t_{i}\right)}=\diamond \quad \text { for all } i \text {. }
$$

Then

$$
\frac{1}{n}\left|z_{[0, n)}\right|_{0} \leq \sum_{i=1}^{\infty} \frac{s_{i}}{t_{i}}=1-\theta
$$

It immediately follows that

$$
B_{n}\left(\mathcal{C}^{(\hat{s}, \hat{t}, k)}\right) \geq k^{\theta n}
$$

and so

$$
h_{\text {top }}\left(\left.\sigma\right|_{\mathcal{C}^{(\hat{s}, \hat{t}, k)}}\right) \geq \lim _{n \rightarrow+\infty} \frac{\log \left(k^{\theta n}\right)}{n}
$$

which ends the proof.

Theorem 31. Let $k$ be a positive integer and let $\hat{s}=\left\{s_{i}\right\}_{i \in \mathbb{N}}, \hat{t}=\left\{t_{i}\right\}_{i \in \mathbb{N}}$ be increasing sequences of positive integers such that $\sum_{i=1}^{\infty} \frac{s_{i}}{t_{i}}<1$. In this case there is no DC1 pair in $\mathcal{C}^{(\hat{s}, \hat{t}, k)}$. 
Proof. It is easy to observe that $0^{\infty}$ is contained in the limit set of any point of $\mathcal{C}^{(\hat{s}, \hat{t}, k)}$. The proof is finished by Lemma 18 and Corollary 15 ,

Theorem 32. Let $k$ be a positive integer and let $\hat{s}=\left\{s_{i}\right\}_{i \in \mathbb{N}}, \hat{t}=\left\{t_{i}\right\}_{i \in \mathbb{N}}$ be increasing sequences of positive integers such that $\sum_{i=1}^{\infty} \frac{s_{i}}{t_{i}}<1$. There exists an uncountable scrambled set of type 2 in $\mathcal{C}^{(\hat{s}, \hat{t}, k)}$.

Proof. The proof is constructive. We start our construction with a construction of the set of parameters. Let us define sequences $S_{n}, M_{n}$ by $S_{1}=1, M_{1}=1$, $S_{n}=2^{n} M_{n-1}$ and $M_{n}=M_{n-1}+S_{n}$. Given $\alpha \in \Sigma^{\omega}$ we define $\check{\alpha} \in \Sigma^{\omega}$ by

$$
\check{\alpha}=\alpha_{1} 0 \alpha_{1} \alpha_{2} 0 \alpha_{1} \alpha_{2} \alpha_{3} \ldots \text {. }
$$

Observe that if $\alpha, \beta \in \Sigma^{\omega}$ and $\alpha \neq \beta$, then there exist increasing sequences of integers $\left\{\nu_{i}\right\}_{i \in \mathbb{N}},\left\{\mu_{i}\right\}_{i \in \mathbb{N}}$ such that

$$
\check{\alpha}_{\nu_{i}}=\check{\beta}_{\nu_{i}} \text { and } \check{\alpha}_{\mu_{i}} \neq \check{\beta}_{\mu_{i}}
$$

for all $i$. As the last auxiliary sequence we define:

$$
\tilde{\alpha}=\check{\alpha}_{1}^{S_{1}} \check{\alpha}_{2}^{S_{2}} \check{\alpha}_{3}^{S_{3}} \ldots \in \Sigma^{\omega} .
$$

Let $z$ be a word over $\{0, \diamond\}$ such that

$$
z_{j}=\diamond \quad \Longleftrightarrow \quad p_{j}^{\left(s_{i}, t_{i}\right)}=\diamond \quad \text { for all } i .
$$

For any $\alpha \in \Sigma^{\omega}$ we define an infinite word $x^{(\alpha)}$ in a way that

$$
x_{j}^{(\alpha)}= \begin{cases}0, & z_{j}=0 \\ \tilde{\alpha}_{j}, & z_{j}=\diamond .\end{cases}
$$

We claim that the set

$$
D=\left\{x^{(\alpha)}: \alpha \in \Sigma^{\omega}\right\} \subset \mathcal{C}^{(\hat{s}, \hat{t}, k)}
$$

is a distributionally scrambled set of type 2 . Let us fix any distinct $\alpha, \beta \in \Sigma^{\omega}$, denote $\theta=1-\sum_{i=1}^{\infty} \frac{s_{i}}{t_{i}}$ and let $\left\{\mu_{i}\right\}_{i \in \mathbb{N}}$ be an increasing sequence such that $\check{\alpha}_{\mu_{i}} \neq$ $\check{\beta}_{\mu_{i}}$ for all $i$. We may assume that $\check{\alpha}_{\mu_{i}}=1$ for all $i$. Observe that

$$
\left|x_{\left[M_{\mu_{i}-1}, M_{\mu_{i}}\right)}^{(\alpha)}\right|_{1} \geq \theta S_{\mu_{i}}
$$

and thus

$$
\xi\left(x^{(\alpha)}, x^{(\beta)}, M_{\mu_{i}}, \frac{1}{2}\right) \leq \lim _{i \rightarrow \infty} \frac{M_{\mu_{i}}-\theta S_{\mu_{i}}}{M_{\mu_{i}}}=1-\lim _{i \rightarrow \infty} \frac{\theta 2^{\mu_{i}}}{2^{\mu_{i}}+1}=1-\theta,
$$

which finally implies that

$$
F_{x^{(\alpha)} x^{(\beta)}}^{*}\left(\frac{1}{2}\right) \leq 1-\theta .
$$

There also exists an increasing sequence $\left\{\nu_{i}\right\}_{i \in \mathbb{N}}$ such that $\check{\alpha}_{\nu_{i}}=\check{\beta}_{\nu_{i}}$ for all $i$. This implies that

and as a result

$$
x_{\left[M_{\nu_{i}-1}, M_{\nu_{i}}\right)}^{(\alpha)}=x_{\left[M_{\nu_{i}-1}, M_{\nu_{i}}\right)}^{(\beta)}=0^{S_{\nu_{i}}}
$$

$$
F_{x^{(\alpha)} x^{(\beta)}}^{*}(t)=1
$$

for any $t>0$. 


\section{INTERVAL MAPS}

Let $s \geq 2$. Recall that an $s$-horseshoe for $f$ consists of closed nondegenerate subintervals $J_{1}, \ldots, J_{s}$ of $I$ with pairwise disjoint interiors and such that $I \subset f\left(J_{i}\right)$ for $i=1, \ldots, s$. An $s$-horseshoe is strong if in addition the intervals $J_{1}, \ldots, J_{s}$ are contained in the interior of $I$ and are pairwise disjoint.

Lemma 33. Let $g: I \rightarrow I$ be a continuous map with a strong $s$-horseshoe $J_{1}, \ldots, J_{s}$ with $s>3$. Then there exists a strong $(s-1)$-horseshoe $I_{1}, \ldots, I_{s-1}$ for $g$ such that $I_{1}$ contains exactly one fixed point.

Proof. Assume that $J_{i}=\left[a_{i}, b_{i}\right]$, where $a_{1}<b_{1}<a_{2}<\cdots<a_{s}<b_{s}$. We may also assume that there exist points $c \leq a_{1}$ and $d \geq b_{s}$ such that for $i=1, \ldots, s$ exactly one of the following two conditions holds: $f\left(a_{i}\right)=c, f\left(b_{i}\right)=d$ or $f\left(a_{i}\right)=d$, $f\left(b_{i}\right)=c$.

Obviously, $J_{1}$ contains at least one fixed point. If there is exactly one fixed point in $J_{1}$, then there is nothing to prove $\left(I_{i}=J_{i}, i=1, \ldots, s-1\right)$. If it does not hold, we have to do some modifications of the given horseshoe. Let us consider the following three possible cases:

(a) $f\left(a_{1}\right)=c$ and $f\left(b_{1}\right)=d$. Let $p$ be a fixed point defined by the formula

$$
p=\sup \left\{x \in J_{1}: g(x)=x\right\} .
$$

It is enough to fix $I_{1}=\left[p, b_{1}\right]$ and $I_{i}=J_{i}$ for $i=2, \ldots, s-1$.

(b) $f\left(a_{2}\right)=c$ and $f\left(b_{2}\right)=d$. It is enough to remove $J_{1}$ to reduce the case (b) to $(a)$.

(c) $f\left(a_{1}\right)=f\left(a_{2}\right)=d$ and $f\left(b_{1}\right)=f\left(b_{2}\right)=c$. Replace $J_{1}$ by the interval $\left[b_{1}, a_{2}\right]$ and remove $J_{2}$ (this way a strong $(s-1)$-horseshoe is produced). Next, repeat the construction from the case (a).

Theorem 34. Let $g: I \rightarrow I$ be a continuous map with $h_{\text {top }}(g)>0$. For any $\varepsilon>0$ there exists a closed and $g$-invariant set $Y^{\varepsilon} \subset I$ such that there is no DC1 pair in $Y^{\varepsilon}$, there exists an uncountable distributionally scrambled set $D$ of type 2 for $\left.f\right|_{Y^{\varepsilon}}$ and

$$
h_{t o p}\left(\left.g\right|_{Y^{\varepsilon}}\right) \geq(1-\varepsilon) h_{t o p}(g) .
$$

Proof. By the Misiurewicz theorem (see e.g [39] for the interval or its generalization 36] for the topological graphs case) there exist sequences $\left\{\iota_{n}\right\}_{n \in \mathbb{N}}$ and $\left\{\kappa_{n}\right\}_{n \in \mathbb{N}}$ such that $g^{\kappa_{n}}$ has a strong $\iota_{n}$-horseshoe and

$$
\limsup _{n \rightarrow+\infty} \frac{1}{\kappa_{n}} \log \iota_{n}=h_{\text {top }}(g) .
$$

By Lemma 33 we may assume that each $\iota_{n}$-horseshoe $J_{1}, \ldots, J_{\iota_{n}}$ has the property that $J_{1}$ contains exactly one fixed point (the limit remains the same if we use $\iota_{n}-1$ instead of $\iota_{n}$ in (34.2) $)$.

It was firstly observed by Louis Block in [12] that in the case of interval maps a horseshoe may be used to obtain a semiconjugacy with a full shift. Later, it became clear that this semiconjugacy may be constructed to be at most two-to-one with the additional property that preimages of periodic points are periodic ([18]). In our case, point $0^{\infty} \in \Sigma_{k}^{\omega}$ is covered exactly one-to-one because the first interval of the horseshoe contains exactly one fixed point (see [21]). 
Let $f=g^{\kappa}$ and let $\Lambda \subset I$ be such that $\pi: \Lambda \rightarrow \Sigma_{\iota}^{\omega}$ is a semiconjugacy with properties described above and such that $\frac{\log \iota}{\kappa}>\sqrt{1-\varepsilon} h_{\text {top }}(g)$.

Let us set any two increasing sequences of integers $\hat{s}=\left\{s_{i}\right\}_{i \in \mathbb{N}}, \hat{t}=\left\{t_{i}\right\}_{i \in \mathbb{N}}$ such that $1-\sum_{i=1}^{\infty} \frac{s_{i}}{t_{i}}>\sqrt{1-\varepsilon}$. We denote $Z^{\varepsilon}=\pi^{-1}\left(\mathcal{C}^{(\hat{s}, \hat{t}, \iota)}\right)$ and

$$
Y^{\varepsilon}=\bigcup_{j=1}^{\kappa} g^{j}\left(Z^{\varepsilon}\right) .
$$

By the well-known properties of semiconjugacy,

$$
h_{t o p}\left(\left.g\right|_{Y^{\varepsilon}}\right) \geq \frac{1}{\kappa} h_{t o p}(f) \geq \sqrt{1-\varepsilon} \frac{\log \iota}{\kappa} \geq(1-\varepsilon) h_{t o p}(g) .
$$

Observe that the assumptions of the theorem guarantee that

$$
\lim _{n \rightarrow+\infty} \operatorname{diam} \pi^{-1}\left(\left\{x \in \Sigma_{k}^{\omega}: x_{[0, n]}=0^{n+1}\right\}\right)=0 .
$$

Then the arguments that there is no $D C 1$ pair in $Y^{\varepsilon}$ are exactly the same as in the proof of Theorem 31. If $D^{\prime} \subset \mathcal{C}^{(\hat{s}, \hat{t}, \iota)}$ is an uncountable $D C 2$ scrambled set for $\sigma$, then it is enough to denote by $D$ a set containing exactly one point from each preimage $\pi^{-1}(x)$, where $x \in D^{\prime}$. The proof that $D$ is the desired distributionally scrambled set of type 2 for $f$ involves exactly the same calculations as the proof of Theorem 32. It is also known [16. Lemma 6] that a $D C 1$ pair for $g^{k}$ remains a $D C 1$ pair for $g$, so the proof is finished.

As we mentioned in the introduction, distributional chaos and positive topological entropy are equivalent notions for continuous maps from a compact interval into itself [52]. It is interesting that this equivalence is no longer valid for subsystems of interval maps.

Theorem 35. Let $g: I \rightarrow I$ be a continuous map with $h_{\text {top }}(g)>0$ and let $\varepsilon>0$. In this case there exist closed and $g$-invariant sets $X, Y \subset I$ such that:

(1) $X \cap Y=\emptyset$,

(2) $h_{\text {top }}\left(\left.g\right|_{X}\right)=0$ and $h_{\text {top }}(g \mid Y) \geq(1-\varepsilon) h_{\text {top }}(g)$,

(3) $\left.g\right|_{X}$ displays uniform distributional chaos and there is no DC1 pair for $\left.g\right|_{Y}$,

(4) there exists a closed set $\Lambda \subset X$ such that $\Lambda$ is $g^{k}$-invariant for some $k>0$ and $\left.g^{k}\right|_{\Lambda}$ exhibits uniform distributional chaos.

Additionally $\left.g\right|_{X}$ is a minimal map and there exists an uncountable distributionally scrambled set $D \subset Y$ of type 2 for $\left.f\right|_{Y}$.

Proof. Applying Theorem 34 we obtain a set $Y$. As we mentioned in the proof of Theorem 34, $g^{k}$ is semiconjugate with a full shift on at least two symbols in a way that the set of points which are not covered one-to-one is at most countable. Then there exist a $g^{k}$-invariant set $\Lambda \subset I$ such that $\Lambda$ is homeomorphic to some $M^{(r)}$ (at least one among the sets of the constructed family is covered exactly one-to-one). We put $X=\bigcup_{i=1}^{k} g^{i}(\Lambda)$. Obviously $X$ remains minimal, $h_{\text {top }}\left(\left.g\right|_{X}\right)=0$ and again by [16. Lemma 6] map $\left.g\right|_{X}$ exhibits uniform distributional chaos.

To finish the proof, observe that if $X \cap Y \neq \emptyset$, then $X \subset Y$; in particular, there are $D C 1$ pairs in $Y$. 


\section{Open questions}

It was proved in [4] that weak mixing does not imply distributional chaos in general (another difference between distributional and Li-Yorke chaos 28]). Corollary 21 implies that it is impossible to construct a minimal system which exhibits dense distributional chaos but is not weakly mixing. A natural question arises:

Question 1. Is it possible to construct a weakly mixing and minimal dynamical system without dense distributional chaos?

One of the possible extensions of dense $\varepsilon$-chaos is the so-called generic $\varepsilon$-chaos (see [48, 59, 11]) where it is assumed that the set of all Li-Yorke pairs for the system forms a residual subset of $X \times X$. In fact, these two notions are equivalent [43; however starting from generic $\varepsilon$-chaos it is easy to find scrambled sets with many additional properties [51. It would be nice to extend this approach to the case of distributional chaos:

Question 2. Are there any mild conditions (weaker than the specification property) which imply dense distributional chaos?

We also remark here that it is impossible to construct a residual scrambled set (and in effect there is no residual distributionally scrambled set) for a map acting on a compact interval. The first proof of this fact was given for extremal Li-Yorke chaos in [14] and next extended on the class of Li-Yorke chaotic maps [23. It is possible to construct maps with scrambled set equal to the whole compacta 27; however such a construction is impossible when $\varepsilon$-scrambled sets are considered 11. From this point of view, it seems to be a rather hard task to construct a map with uniform distributional chaos and residual distributionally scrambled set, provided it exists. The answer for Question 2 may be the first step towards the solution.

According to the author's knowledge, there exist only two papers [58, 47] with examples of positive topological entropy systems with no distributionally chaotic pairs of type 1. In both examples an uncountable distributionally scrambled set of type 2 may be obtained, despite the fact that the main ideas behind these examples are different (the first is two-dimensional, and the second is zero-dimensional). We obtain another open problem:

Question 3. Does positive topological entropy imply the existence of a distributionally chaotic pair of type 2 ?

\section{ACKNOWLEDGEMENTS}

The author would like to thank Prof. L. Snoha for providing him with a copy of [28. Relations between proximality and $D C 1$, in particular Corollary 15, were suggested to the author by the (unknown) reviewer.

An example of a proximal map with positive topological entropy was probably known many years before 24] (where it is only a step in a more complex construction); however it seems that nobody remembers the reference. The author thanks Professors F. Blanchard, T. Downarowicz, E. Glasner and X. Ye for their help during his (unsuccessful) search for that old article.

This research was supported by the project No. NN201 272333 for the years 2007-2009 from the Polish Ministry of Science and Higher Education, an annual 
national scholarship for young scientists from the Foundation for Polish Science and AGH grant No. 10.420.03.

\section{REFERENCES}

[1] E. Akin and S. Kolyada, Li-Yorke sensitivity, Nonlinearity, 16 (2003) 1421-1433. MR1986303 (2004c:37016)

[2] L. Alseda, J. Llibre and M. Misiurewicz, Combinatorial dynamics and entropy in dimension one. Advanced Series in Nonlinear Dynamics, World Scientific Publishing Co., River Edge, NJ, 1993. MR1255515 (95j:58042)

[3] J. Auslander, Minimal flows and their extensions, North-Holland Mathematics Studies, 153. North-Holland Publishing Co., Amsterdam, 1988 MR956049 (89m:54050)

[4] J. Auslander and J. Yorke, Interval maps, factors of maps and chaos, Tohoku Math. J., 32 (1980) 177-188. MR:580273 (82b:58049)

[5] M. Babilonová-S̆tefánková, Extreme chaos and transitivity, Internat. J. Bifur. Chaos Appl. Sci. Engrg., 13 (2003) 1695-1700. MR 2015619 (2004i:37075)

[6] F. Balibrea, L. Reich and J. Smítal, Iteration theory: Dynamical systems and functional equations, Dynamical systems and functional equations (Murcia, 2000), Internat. J. Bifur. Chaos Appl. Sci. Engrg., 13 (2003), 1627-1647. MR2015613 (2004m:37014)

[7] F. Balibrea, B. Schweizer, A. Sklar, and J. Smítal, Generalized specification property and distributional chaos, Internat. J. Bifur. Chaos 13 (2003), 1683-1694. MR2015618|(2004m:37015)

[8] F. Balibrea, J. Smítal, and M. Štefánková, The three versions of distributional chaos, Chaos Solitons Fractals 23 (2005), 1581-1583. MR2101573 (2005f:37040)

[9] J. Banks, Topological mapping properties defined by digraphs, Discrete Contin. Dynam. Systems, 5 (1999) 83-92. MR1664461 (99j:54038)

[10] F. Blanchard, E. Glasner, S. Kolyada and A. Maass, On Li-Yorke pairs, J. Reine Angew. Math. 547 (2002) 51-68. MR.1900136 (2003g:37014)

[11] F. Blanchard, W. Huang and L. Snoha, Topological size of scrambled sets, Colloq. Math., 110 (2008) 293-361. MR2353910 (2008j:37013)

[12] L. Block, Homoclinic points of mappings of the interval, Proc. Amer. Math. Soc. 72 (1978), 576-580. MR509258 (81m:58063)

[13] R. Bowen, Topological entropy and axiom A, in "Global Analysis", Proceedings of Symposia on Pure Mathematics, vol. 14, Amer. Math. Soc., Providence, 1970, pp. 23-41. MR0262459 $(41: 7066)$

[14] A. M. Bruckner and T. Hu, On scrambled sets for chaotic functions, Trans. Amer. Math. Soc., 301 (1987) 289-297. MR879574 (88f:26003)

[15] Z. Chen, G. Liao and L. Wang, The complexity of a minimal sub-shift on symbolic spaces, J. Math. Anal. Appl., 317 (2006), 136-145. MR2205317 (2007j:37021)

[16] Z. Chu, X. Duan, G. Liao and L. Wang, The set of recurrent points of a continuous selfmap on an interval and strong chaos, J. Appl. Math. \& Computing 14 (2004), 277 - 288. MR2025438 (2004j:37069)

[17] J. P. Clay, Proximity relations in transformation groups, Trans. Amer. Math. Soc., 108 (1963) 88-96. MR0154269 (27:4218)

[18] W. A. Coppel, Maps of an interval, IMA Preprint Series, vol. 26.

[19] M. Denker, C. Grillenberger, and K. Sigmund, Ergodic theory on compact spaces, SpringerVerlag, Berlin, 1976. MR0457675 (56:15879)

[20] R. Devaney, A first course in chaotic dynamical systems, Perseus Books, 1992. MR1202237 (94a:58124)

[21] B.-S. Du, On the invariance of Li-Yorke chaos of interval maps, J. Difference Equ. Appl., 11 (2005) 823-828. MR2159799 (2006d:37063)

[22] V.V. Fedorenko, A.N. Sharkovskij and J. Smítal, Characterizations of weakly chaotic maps of the interval, Proc. Amer. Math. Soc., 110 (1990) 141-148. MR1017846 (91a:58148)

[23] T. Gedeon, There are no chaotic mappings with residual scrambled sets, Bull. Austral. Math. Soc, 36 (1987), 411-416. MR923822 (89d:58083)

[24] E. Glasner and B. Weiss, Sensitive dependence on initial conditions, Nonlinearity, 6 (1993) 1067-1075. MR1251259(94j:58109)

[25] S. Glasner, Compressibility properties in topological dynamics, Amer. J. Math., 97 (1975) 148-171. MR0365537(51:1789) 
[26] _ Proximal flows, Lecture Notes in Mathematics, Vol. 517. Springer-Verlag, Berlin-New York, 1976. MR0474243 (57:13890)

[27] W. Huang and X. Ye, Homeomorphisms with the whole compacta being scrambled sets, Ergodic Theory Dynam. Systems, 21 (2001), 77-91. MR1826661 (2002d:37020)

[28] A. Iwanik, Independence and scrambled sets for chaotic mappings, The mathematical heritage of C. F. Gauss, 372-378, World Sci. Publ., River Edge, NJ, 1991. MR1146241 (92k:58176)

[29] W. Huang and X. Ye, Devaney's chaos or 2-scattering implies Li-Yorke chaos, Topology Appl. 117 (2002), 259-272. MR1874089(2003b:37017)

[30] K. Janková and J. Smítal, A characterization of chaos, Bull. Aust. Math. Soc., 34 (1986) 283-292. MR854575 (87k:58178)

[31] I. Kan, A chaotic function possessing a scrambled set with positive Lebesgue measure, Proc. Amer. Math. Soc., 92 (1984) 45-49. MR749887 (86b:26009a)

[32] S. F. Kolyada, Li-Yorke sensitivity and other concepts of chaos, Ukrainian Math. J. 56 (2004), 1242-1257. MR2136308 (2005k:37028)

[33] S. Li, $\omega$-chaos and topological entropy, Trans. Amer. Math. Soc. 339 (1993), 243-249. MR.1108612 (93k:58153)

[34] T. Y. Li and J. A. Yorke, Period three implies chaos, Amer. Math. Monthly 82 (1975), 985-992. MR0385028(52:5898)

[35] G. Liao and L. Wang, Almost periodicity and distributional chaos. Foundations of computational mathematics (Hong Kong, 2000), 189-210, World Sci. Publ., River Edge, NJ, 2002 MR.2021982 (2004m:37016)

[36] J. Llibre and M. Misiurewicz, Horseshoes, entropy and periods for graph maps, Topology 32 (1993), 649-664. MR1231969 (94k:58113)

[37] R. Mañé, Ergodic theory and diferentiable dynamics, Springer-Verlag, Berlin, 1987. MR889254 (88c:58040)

[38] M. Martelli, M. Dang and T. Seph, Defining Chaos, Math. Mag., 71 (1998) 112-122. MR1706086 (2000g:37001)

[39] M. Misiurewicz, Horseshoes for mappings of the interval, Bull. Acad. Pol. Sci., Sér. Sci. Math., 27 (1979) 167-169. MR.542778 (81b:58033)

[40] _ Chaos almost everywhere, Iteration theory and its functional equations, Proc. Int. Symp., Schloß Hofen (Lochau)/Austria 1984, Lect. Notes Math., 1163 (1985) 125-130. MR829765 (87e:58152)

[41] M. Morse and G. A. Hedlund, Symbolic Dynamics, Amer. J. Math., 60 (1938) 815-866. MR.1507944

[42] Symbolic dynamics. II. Sturmian trajectories, Amer. J. Math., 62 (1940) 1-42 . MR0000745 (1:123d)

[43] E. Murinová, Generic chaos in metric spaces, Acta Univ. M. Belii Ser. Math., 8 (2000) 43-50. MR.1857201 (2002h:37017)

[44] P. Oprocha, Relations between distributional and Devaney chaos, Chaos, 16 (2006) 033112. MR2265261 (2007g:37009)

[45] _ Specification properties and dense distributional chaos, Discrete Contin. Dyn. Syst., 17 (2007) 821-833. MR2276477 (2008a:37022)

[46] P. Oprocha and P. Wilczyñski, Shift spaces and distributional chaos, Chaos, Solitons and Fractals, 31 (2007) 347-355. MR.2259760 (2007i:37019)

[47] R. Pikuła, On some notions of chaos in dimension zero, Colloq. Math., 107 (2007), 167-177 MR.2284159 (2007m:37021)

[48] J. Piórek, On the generic chaos in dynamical systems, Univ. Iagel. Acta Math., 25 (1985) 293-298. MR837847 (87h:58131)

[49] D. Ruelle and F. Takens, On the nature of turbulence, Commun. Math. Phys., 20 (1971) 167-192. MR0284067 (44:1297)

[50] S. Ruette, Dense chaos for continuous interval maps, Nonlinearity, 18 (2005) 1691-1698. MR2150350 (2006c:37038)

[51] B. Schweizer, A. Sklar and J. Smítal, Distributional (and other) chaos and its measurement, Real. Anal. Exch., 27 (2001/2002) 495-524. MR1844132 (2002c:37056)

[52] B. Schweizer and J. Smítal, Measure of chaos and a spectral decomposition of dynamical systems on the interval, Trans. Amer. Math. Soc. 344 (1994), 737-754. MR.1227094(94k:58091)

[53] A. Sklar and J. Smítal, Distributional chaos on compact metric spaces via specification properties, J. Math. Anal. Appl. 241 (2000), 181-188. MR.1739200(2000k:37021) 
[54] J. Smítal, A chaotic function with some extremal properties, Proc. Amer. Math. Soc., 87 (1983) 54-56. MR677230 (84h:26008)

[55] _ A chaotic function with a scrambled set of positive Lebesgue measure, Proc. Amer. Math. Soc., 92 (1984) 50-54. MR749888 (86b:26009b)

[56] Chaotic functions with zero topological entropy, Trans. Amer. Math. Soc., 297 (1986) 269-282. MR849479 (87m:58107)

[57] — Various notions of chaos, recent results, open problems, Various notions of chaos, recent results, open problems. Report on the Summer Symposium in Real Analysis XXVI. Real Anal. Exchange 2002, 26th Summer Symposium Conference, suppl., 81-85 MR2182670

[58] J. Smítal and M. Stefánková, Distributional chaos for triangular maps. Chaos, Solitons and Fractals 21 (2004) 1125-1128. MR2047330 (2005a:37017)

[59] L. Snoha, Generic chaos, Comment. Math. Univ. Carol., 31 (1990) 793-810. MR.1091377 (92b:58149)

[60] , Dense chaos, Comment. Math. Univ. Carol., 33 (1992) 747-752. MR.1240197 (94i:58128)

[61] S. Wiggins, Chaotic transport in dynamical systems, Interdisciplinary Applied Mathematics, Springer-Verlag, New York, 1992 MR:1139113 (93c:58152)

Faculty of Applied Mathematics, AGH University of Science and Technology, al. Mickiewicza 30, 30-059 Kraków, Poland

E-mail address: oprocha@agh.edu.pl 